ORIGINAL ARTICLE

\title{
Frequency of Hypoalbuminemia in Acute Ischemic Stroke patients Based on Stroke Severity
}

\author{
EHSAN UL HAQ ${ }^{1}$, ALI QAYYUM ${ }^{2}$, HAFIZ AMMAR QAYYUM ${ }^{3}$, MUNASHRA ANAM ${ }^{4}$, ABDUL ROUF KHAN ${ }^{5}$, KAMRAN $^{2}$ \\ AHMED $^{6}$, FUAD SHAFIQ7 \\ ${ }^{1}$ Senior Registrar Neurology, Al Sabah Hospital, Kuwait \\ ${ }^{2}$ Senior Registrar Neurology, Central Park Teaching Hospital Lahore \\ ${ }^{3}$ Senior Physiotherapist, Ittefaq Hospital and Bahria Hospital, Lahore \\ ${ }^{4}$ Medical Officer, Cantonment General Hospital Lahore, Cantt \\ ${ }^{5}$ Post Graduate Trainee (Neurology) JPMC, Karachi \\ ${ }^{6}$ House Officer Benazir hospital, Rawalpindi \\ ${ }^{7}$ Prof. of Medicine Department, Central Park Teaching Hospital, Lahore \\ Correspondence to Dr. Ali Qayyum, Email: aliqayyum211@hotmail.com, Phone: 03094361017
}

\begin{abstract}
Background: Stroke is a serious public health issue and third leading cause of death worldwide. Hypoalbuminemia is commonly found factor in patients of stroke and is also associated with severe disease as well as pro inflammatory patterns of serum protein electrophoresis. Therefore, further research for understanding the role of Hypoalbuminemia in stroke is important to devise strategies for better management of stroke.

Aim : To determine the frequency of hypoalbuminemia in acute ischemic stroke patients based on stroke severity. Methods: This descriptive cross- sectional study was conducted in Shifa International hospital stroke unit for 6 months from May 15, 2018 till Nov 15, 2018. Data was collected from 100 patients using purposive sampling. After taking consent from patient or attendant, the demographic data was collected on a structured proforma. Baseline serum albumin and stroke severity using the NIHSS score was also assessed. All data was entered and analysed using SPSS 21. After descriptive analysis, post stratified Chi Square test was applied for gender and age categories.

Results: The mean age of patients was $63.60 \pm 11.87$ years with $57(57 \%)$ male and $43(43 \%)$ female cases. The mean serum albumin level was $4.03 \pm 0.94$ with minimum and maximum values as 1.50 and 5.5 . Among cases with minor, moderately severe and with severe stroke, $6(37.5 \%)$ cases, $18(25.7 \%)$ cases and $6(42.9 \%)$ cases had Hypoalbuminemia. The frequency of hypoalbuminemia was statistically same with respect to severity of stroke, $\mathrm{p}$ value $>0.05$.

Conclusion: This study concludes that the frequency of hypoalbuminemia in acute ischemic stroke patients was diagnosed in almost one third cases, however, no statistical association could be found. Hence, screening for hypoalbuminemia should be done for better management of stroke patients.

Keywords: Storke, NIHSS score, serum albumin, hypoalbuminemia, mortality
\end{abstract}

\section{INTRODUCTION}

Stroke is a critical public health issue with high prevalence and worse health outcomes ${ }^{1}$. It is second highest cause of mortality globally2. At least 16.9 million individuals are reported to experience stroke for the first time in life. ${ }^{3}$ Moreover, stroke has been a direct cause of almost 6 million deaths with increasing incidence of $68 \%, 84 \%, 26 \%$, and $12 \%$ respectively with highest reported percentages in developing and under developed countries. ${ }^{4}{ }^{5}$ More than $85 \%$ strokes are Ischemic and their increasing annual incidence has alarmed the global health sector. The prevalence and severity of stroke has burdened the already compromised healthcare systems of developing and under developed countries $^{6}$. A number of genetic, biological, environmental, non-modifiable and modifiable risk factors are responsible for stroke ${ }^{7}, 8$.

Stroke is a disease caused by multifactorial facets. One of these factors is hypoalbuminemia ${ }^{9}$. Any fluctuations in the levels of serum albumin can severely affect the course of disease, its severity and outcome ${ }^{10}$. Association of hypoalbuminemia and stroke severity has been

Received on 13-01-2021

Accepted on 13-05-2021 described in many studies ${ }^{11,12}$. A normal serum albumin level is not only associated with good outcome ${ }^{13}$ but can also reduce the risk of in hospital mortality. Similarly, low level of serum albumin can serve as a predictor for recurring stroke, in hospital deaths, infections and other complications in stroke patients. ${ }^{14}$ Moreover, low level of albumin in the blood has been reported to have poor prognosis in intra-cerebral hemorrhage as well. ${ }^{15,} 16$ Also, Severity of ischemic stroke worsens with hypoabluminemia in the stroke patients ${ }^{17}$.

One study enrolled 112 Acute Ischemic Stroke (AIS) patients consecutively to find association between hospital outcome and hypoalbuminemia among AIS patients. They reported that $43(87.7 \%)$ of 49 patients who expired in hospital were hypoalbumenic. They also reported that association between hypoalbumenia and AIS was significantly higher in non-survivors (34.5 vs. $16.9 \& p=$ 0.001 vs 0.04 respectively) ${ }^{18}$.

The percentage of hypoalbuminemia among patients with AIS is published as $41.6 \%$ \& $42 \%$ in Lahore and KPK respectively. ${ }^{19,20}$ However, no local study has assessed the hypoalbuminemia with respect to severity of stroke disease. Hence, this study aims to find association between hypoalbuminemia and severity of stroke in AIS 
patients. The results of this study can lead towards early screening of stroke patients for their serum albumin levels and timely management of high risk patients.

\section{MATERIAL AND METHODS}

This descriptive cross- sectional study was conducted in Shifa International hospital (stroke unit) for 6 month [May 15, 2018 till Nov 15, 2018]. The data was collected through consecutive sampling from sample of 100 participants calculated using WHO sample size calculator keeping confidence level at $95 \%$, prevalence of hypoalbuminemia as $16.7 \%{ }^{17}$ and absolute precision of $8 \%$. Patients with evidence of ischemic stroke on MRI or CT scan aged 18-80 years of both genders and admitted to stroke unit within 72hours of onset of stroke symptom were included in the study. Patients with Transient ischemic attack by taking history, having Intra cerebral bleed, decompensated liver disease clinically by signs of disease like abdominal distension, jaundice etc, nephrotic syndrome clinically by pedal and periorbital edema, protein losing enteropathy by taking history of chronic diarrhea were all excluded from study. The research was approved by Ethical Review Board.

After taking Informed consent from patients, sociodemographic and clinical information was taken on a structured proforma. Information about baseline serum and severity of stroke using NIHSS score was also taken. Data was entered and analyzed using SPSS v. 21. Frequency and percentage was calculated for qualitative variables like type of stroke, hypoalbuminemia, gender, and severity of stroke, whereas, mean and SD was calculated for quantitative variables like age and albumin level. Frequency of hypoalbuminemia in different severity of ischemic stroke was calculated and compared by chi square test. Effect modifiers like age, gender were controlled through stratification. Post stratification chi square test was applied. $\mathrm{P}$ - value $<0.05$ was considered significant.

\section{RESULTS}

The mean age of patients was $63.60 \pm 11.87$ years with minimum and maximum age as 33 and 80 years. There were $35(35 \%)$ cases aged $<60$ years and $65(65 \%)$ cases were $60-80$ years old. There were $57(57 \%)$ male and $43(43 \%)$ female cases with higher male to female ratio. A total of $80(80 \%)$ cases had anterior circulation and $20(20 \%)$ cases had posterior circulation. A total of $16(16 \%)$ cases had minor, $70(70 \%)$ cases had moderately severe stroke and $14(14 \%)$ cases had severe stroke. The mean serum albumin level was $4.03 \pm 0.94$ with minimum and maximum values as 1.50 and 5.5 . A total of $30(30 \%)$ cases had hypoalbuminemia while $70(70 \%)$ cases had normal levels. Among cases with minor, moderately severe and with severe stroke, $6(37.5 \%)$ cases, $18(25.7 \%)$ cases and $6(42.9 \%)$ cases had Hypoalbuminemia. The frequency of hypoalbuminemia was statistically same with respect to severity of stroke, $p$-value $>0.05$. In age group of $<60$ years, Among cases with minor, moderately severe and with severe stroke 2(40\%), 6(24\%) and 2(40\%) cases had hypoalbuminemia while in cases aged $60-80$ years, $10(28.6 \%), 4(36.4 \%)$ and $4(44.4 \%)$ cases had Hypoalbuminemia. The frequency of hypoalbuminemia was statistically same with respect to severity of stroke, in both age groups, $p$-value $>0.05$. In male patients, among cases with minor, moderately severe and with severe stroke $3(33.3 \%)$, $11(27.5 \%)$ and $3(37.5 \%)$ cases had hypoalbuminemia while in female patients, 3(42.9\%), $7(23.3 \% \%)$ and $3(50 \%)$ cases had Hypoalbuminemia. The frequency of hypoalbuminemia was statistically same with respect to severity of stroke, in genders, $p$-value $>0.05$. Moreover, the frequency of hypoalbuminemia was statistically same with respect to severity of stroke, in different types of stroke, $p$-value $>0.05$.

Table-1: Descriptive statistics of age (years) and Serum albumin

\begin{tabular}{|l|c|c|}
\hline & Age (years) & Serum albumin \\
\hline Mean & 63.60 & 4.03 \\
\hline S.D & 11.87 & 0.94 \\
\hline Range & 47.00 & 4.00 \\
\hline Minimum & 33.00 & 1.50 \\
\hline Maximum & 80.00 & 5.50 \\
\hline
\end{tabular}

Table-2: Frequency distribution of gender, type of stroke, severity of stroke and hypoalbuminemia

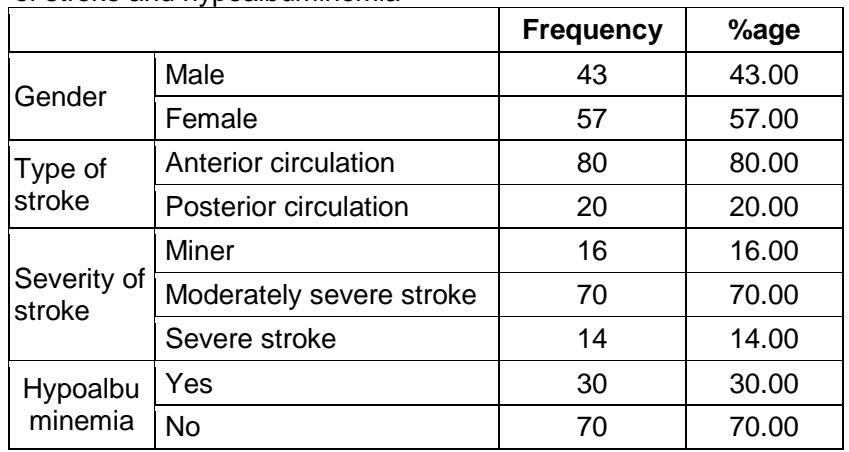

Fig-1: Distribution of age groups (years)

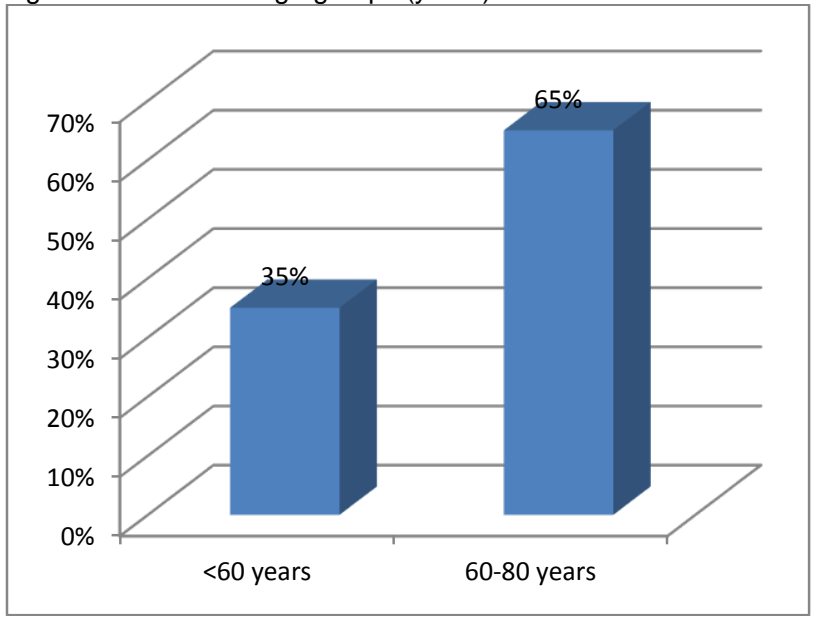


Hypoalbuminemia in Acute Ischemic Stroke patients

Table-3: Comparison of severity of stroke, anterior circulation, posterior circulation in hypoalbuminemia

\begin{tabular}{|c|c|c|c|c|c|}
\hline & \multicolumn{2}{|c|}{ Hypoalbuminemia } & \multirow{2}{*}{ Chi-square } & \multirow{2}{*}{ p-value } \\
\hline & & Yes & No & & \\
\hline \multirow{3}{*}{ Severity of stroke } & Minor & $6(37.5 \%)$ & $10(62.5 \%)$ & \multirow{3}{*}{2.143} & \multirow{3}{*}{0.343} \\
\hline & Moderately severe stroke & $18(25.9 \%)$ & $52(74.3 \%)$ & & \\
\hline & Severe stroke & $6(42.9 \%)$ & $8(57.1 \%)$ & & \\
\hline \multirow{3}{*}{ Anterior circulation } & Minor & $4(36.4 \%)$ & $7(63.6 \%)$ & \multirow{3}{*}{1.216} & \multirow{3}{*}{0.544} \\
\hline & Moderately severe stroke & $17(30.4 \%)$ & $39(69.6 \%)$ & & \\
\hline & Severe stroke & $6(46.2 \%)$ & $7(53.8 \%)$ & & \\
\hline \multirow{3}{*}{ Posterior circulation } & Minor & $2(40 \%)$ & $3(60 \%)$ & \multirow{3}{*}{3.305} & \multirow{3}{*}{0.192} \\
\hline & Moderately severe stroke & $1(7.1 \%)$ & $13(92.9 \%)$ & & \\
\hline & Severe stroke & $0(0 \%)$ & $1(100.0 \%)$ & & \\
\hline
\end{tabular}

Table-4: Comparison of severity of stroke in hypoalbuminemia with respect to age groups

\begin{tabular}{|c|c|c|c|c|c|}
\hline \multirow{2}{*}{ Age Groups (years) } & \multirow{2}{*}{ Severity of stroke } & \multicolumn{2}{|c|}{ Hypoalbuminemia } & \multirow{2}{*}{ Chi-square } & \multirow{2}{*}{$p$-value } \\
\hline & & Yes & No & & \\
\hline \multirow{3}{*}{$<60$ Years } & Minor & $2(40.0 \%)$ & $3(60.0 \%)$ & \multirow{3}{*}{0.896} & \multirow{3}{*}{0.639} \\
\hline & Moderately severe stroke & $6(24.0 \%)$ & $19(76.0 \%)$ & & \\
\hline & Severe stroke & $2(40 \%)$ & $3(60.0 \%)$ & & \\
\hline \multirow{3}{*}{$60-80$ Years } & Minor & $4(36.4 \%)$ & $7(63.6 \%)$ & \multirow{3}{*}{1.307} & \multirow{3}{*}{0.520} \\
\hline & Moderately severe stroke & $12(26.7 \%)$ & $33(73.3 \%)$ & & \\
\hline & Severe stroke & $4(44.4 \%)$ & $5(55.6 \%)$ & & \\
\hline
\end{tabular}

Table-5: Comparison of Severity of stroke in Hypoalbuminemia with respect to gender

\begin{tabular}{|c|c|c|c|c|c|}
\hline \multirow{2}{*}{ Gender } & \multirow{2}{*}{ Severity of stroke } & \multicolumn{2}{|c|}{ Hypoalbuminemia } & \multirow{2}{*}{ Chi-square } & \multirow{2}{*}{ p-value } \\
\hline & & Yes & No & & \\
\hline \multirow{3}{*}{ Male } & Minor & $3(33.3 \%)$ & $6(66.7 \%)$ & \multirow{3}{*}{0.381} & \multirow{3}{*}{0.826} \\
\hline & Moderately severe stroke & $11(27.5 \%)$ & $29(72.5 \%)$ & & \\
\hline & Severe stroke & $3(37.5 \%)$ & $5(62.5 \%)$ & & \\
\hline \multirow{3}{*}{ Female } & Minor & $3(42.9 \%)$ & $4(57.1 \%)$ & \multirow{3}{*}{2.317} & \multirow{3}{*}{0.314} \\
\hline & Moderately severe stroke & $7(23.3 \%)$ & $23(76.7 \%)$ & & \\
\hline & Severe stroke & $3(50 \%)$ & $3(50 \%)$ & & \\
\hline
\end{tabular}

\section{DISCUSSION}

Stroke remains one of the leading causes of morbidity and mortality worldwide. The severity and deaths due to stroke are even more in developing and under developed countries. $^{21}$ Stroke is clinically defined as neurological deficit caused by problems in local vascular system. Stroke is broadly divided into two types, Ischemic and hemorrhagic. ${ }^{22}$ Ischemic stroke occurs because of decreased blood supply to brain and hemorrhagic stroke occurs because of bleeding. ${ }^{23}$. Hence due to malfunctioning in brain and other body organs stroke occurs. Almost 15 million individuals are estimated to experience stroke each year with majority of disease burden in developing countries, chiefly because of adaptation of westernized and stagnant lifestyles and more consumption of fast foods 2425 .

Some common symptoms of stroke are poor senses and motor abilities in one side of the body, nervousness, dysarthria, loss of sight and vertigo. Severe headache is also reportedly associated with stroke ${ }^{26}$. These symptoms usually appear shortly after occurrence of stroke. In Transient Ischemic Attack (TIA), these symptoms disappear usually after a couple of hours. But the confirmatory diagnosis can only be done through CT scan or MRI. An early diagnosis is imperative in management of stroke timely with medication. Many countries manage stroke patients and help in recovering the lost function of patients in specially designated stroke units ${ }^{27}$. The stroke severity on neurologic examination is typically the most important factor that affect short- and long-term outcome ${ }^{28}$.

Literature reports that any change in serum albumin level may considerably effect the health outcomes of stroke patients. Serum albumin levels have many roles normal body function of body, one of which is neuroprotective role ${ }^{29}$. In current study, 30(30\%) cases had hypoalbuminemia whereas, $70(70 \%)$ cases had normal levels of serum albumin. In another study, hypoalbuminemia was found in $45.5 \%$ of patients ${ }^{30}$, that is high frequency when compared with results of current study. Contrary to this, another study reported low prevalence of hypoalbuminemia i.e. $16.7 \%$ as compared to current study ${ }^{17}$. In Pakistan, the frequency of hypoalbuminemia in acute ischemic stroke patients has been reported as high as $41.6 \%$ and $42 \%$ in Lahore and KPK, respectively ${ }^{19,20}$. These findings are also comparable with current study. 
We actually compared albumin levels with outcome, but no association was found with severity of stroke i.e. among cases with minor, moderately severe and with severe stroke, $6(37.5 \%)$ cases, $18(25.7 \%)$ cases and $6(42.9 \%)$ cases had Hypoalbuminemia respectively. The frequency of hypoalbuminemia was statistically same with respect to severity of stroke, $p$-value $>0.05$. Another study reported that severity of ischemic stroke worsens with hypoabluminemia ${ }^{17}$. Yet another study showed that $80 \%$ patients with severe stroke had hypoalbumineamia vs 28.5 $\%$ among patients with moderate stroke and $16.7 \%$ with mild ischemic stroke ${ }^{17}$.

Another study reported mean albumin level among patients of stroke with poor outcome as $2.02 \pm 0.67 \mathrm{gm} / \mathrm{dl}$ whereas $3.08 \pm 0.61 \mathrm{gm} / \mathrm{dl}$ among patients with good outcomes $^{13}$. Low level of serum albumin also serve as predictor of recurring stroke ${ }^{14}$ as well as poor clinical outcomes for intra- cerebral hemorrhage ${ }^{15,16}$.

Serum albumin level shows neuroprotective effect due to its functions related to endothelium, the venular perfusion and anti oxidant qualities ${ }^{31}$. Therefore, a number of studies report lower levels of serum albumin levels have significant association with in hospital mortality and other poor outcomes ${ }^{32}$. Moreover, the stroke patients having hypoalbuminemia have also more frequent infective complications and severe disease. Other studies have also found that average levels of serum albumins were not only significantly lower among stroke patients compared to the controls, but it was also considerably lower among the patients who died or were physically dependent at discharge time ${ }^{33}$.

Conclusively, hypoalbuminemia is commonly observed in patients of ischemic stroke and is correlated with poor clinical outcomes and in hospital mortality. Therefore patients should be screened for serum albumin levels at time of admission and those with low levels should be considered high risk and managed accordingly.

\section{Conflict of interest: None}

\section{CONCLUSION}

This study concludes that the frequency of hypoalbuminemia in acute ischemic stroke patients was found in almost one third cases, i.e. $30 \%$ but its association could not be established with stroke severity. So, patients presenting with stroke must be evaluated for hypoalbuminemia so that appropriate management can be ensured. By correcting hypoalbuminemia the related morbidity and mortality can be reduced. As Hypoalbuminemia in acute stroke patients is associated with increased mortality and morbidity.

Author's contribution: EH: Design, Conceived the original data, Theoretical Formulation, Analytical Calculation, Manuscript Writing, AQ: Design ,Theoretical formulation, Analytical Calculation, Manuscript Writing , HAQ: Design, Analytical Calculation, Manuscript Writing, MA: Analytical Calculation, Manuscript Writing, Plagiarism Removal, ARK: Analytical Calculation, Manuscript Writing, KA: Analytical Calculation, Manuscript Writing, Final Write Up, FS: Design, Literature Search, Final Read Up

Conflict of Interest: There is no conflict of interest

\section{REFERENCES}

1. Zhou $H$, Wang A, Meng $X$, Lin J, Jiang $Y$, Jing J, et al. Low serum albumin levels predict poor outcome in patients with acute ischaemic stroke or transient ischaemic attack. Stroke and Vascular Neurology. 2021:svn-2020-000676.

2. Healey JS, Oldgren J, Ezekowitz M, Zhu J, Pais P, Wang J, et al. Occurrence of death and stroke in patients in 47 countries 1 year after presenting with atrial fibrillation: a cohort study. The Lancet. 2016;388(10050):1161-9.

3. Alhazzani A, Mahfouz A, Abolyazid A, Awadalla N, Aftab R, Faraheen $A$, et al. Study of stroke incidence in the aseer region, Southwestern Saudi Arabia. Int J Environ Res Public Health. 2018;15(2):1-7.

4. Feigin VL, Forouzanfar MH, Krishnamurthi R, Mensah GA, Connor M, Bennett DA, et al. Global and regional burden of stroke during 1990-2010: findings from the Global Burden of Disease Study 2010. The Lancet. 2014;383(9913):245-55.

5. Cabral NL, Freire AT, Conforto AB, dos Santos N, Reis FI, Nagel $V$, et al. Increase of stroke incidence in young adults in a middle-income country: a 10-year population-based study. Stroke. 2017;48(11):2925-30.

6. Abraham A, Burrows S, Abraham NJ, Mandal B. Modified frailty index and hypoalbuminemia as predictors of adverse outcomes in older adults presenting to acute general surgical unit. Revista espanola de geriatria y gerontologia. 2020;55(2):70-5.

7. Vanacker P, Faouzi M, Eskandari A, Maeder P, Meuli R, Michel P. Large arterial occlusive strokes as a medical emergency: need to accurately predict clot location. Eur $\mathrm{J}$ Emerg Med. 2017;24(5):353-8.

8. Edwards JD, Kapral MK, Fang J, Swartz RH. Long-term morbidity and mortality in patients without early complications after stroke or transient ischemic attack. CMAJ. 2017;189(29):E954-E61.

9. Gao J, Zhao Y, Du M, Guo H, Wan T, Wu M, et al. Serum Albumin Levels and Clinical Outcomes Among Ischemic Stroke Patients Treated with Endovascular Thrombectomy. Neuropsychiatric Disease and Treatment. 2021;17:401.

10. Babu MS, Kaul S, Dadheech S, Rajeshwar K, Jyothy A, Munshi A. Serum albumin levels in ischemic stroke and its subtypes: correlation with clinical outcome. Nutrition. 2013;29(6):872-5.

11. Baby P. Malnutrition in acute stroke: what are we treating? British Journal of Neuroscience Nursing. 2020;16(Sup2):S4S9.

12. Purnama MI, Hasan Machfoed M, Eko Wahono R J. Association of Early Nutritional Status with the Clinical Severity in Patients with Acute Stroke. Indian Journal of Forensic Medicine \& Toxicology. 2020;14(2).

13. Abubakar S, Sabir A, Ndakotsu M, Imam M, Tasiu M. Low admission serum albumin as prognostic determinant of 30 day case fatality and adverse functional outcome following acute ischemic stroke. Pan Afr Med J. 2013;14(1):1-7.

14. Zhang Q, Lei $Y-X$, Wang $Q$, Jin $Y-P$, Fu R-L, Geng $H-H$, et al. Serum albumin level is associated with the recurrence of acute ischemic stroke. Am J Emerg Med. 2016;34(9):18126.

15. Morotti A, Marini S, Lena UK, Crawford K, Schwab K, Kourkoulis C, et al. Significance of admission hypoalbuminemia in acute intracerebral hemorrhage. J Neurol. 2017;264(5):905-11.

16. Behrouz R, Godoy DA, Topel CH, Birnbaum LA, Caron J-L, Grandhi R, et al. Early hypoalbuminemia is an independent predictor of mortality in aneurysmal subarachnoid hemorrhage. Neurocrit Care. 2016;25(2):230-6.

17. James R, Antony J, Sreedhar S, Mathew R, Surendran A. Study of serum albumin as a predictor of short-term functional outcome in acute ischaemic stroke. J Evolution Med Dental Sci. 2017;6(36):2957-63. 
18. Vahedi A, Lotfinia I, Sad R, Halimi M, Baybordi $H$ Relationship between admission hypoalbuminemia and inhospital mortality in acute stroke. Pakistan Journal of Biological Sciences. 2011;14(2):118-22.

19. Butt A, Ali MR, Zameer H. Frequency of Hypoalbuminemia and Mortality in Acute Ischemic Stroke. Hospitals. 2016;20:1-3.

20. Javid RA, Bhatti A, Azhar MA. Frequency of Hypoalbuminemia in patients with Ischemic Stroke. PJMHS. 2016;10:571-3.

21. Aboud HN, Mohammed HA, Kamil MM, Hassan B. The Significance of Hypoalbumineamia and Hypoproteinemia in Patients with Stroke. Neuroscience and Medicine. 2018;9(3):105-15.

22. Di Napoli M, Behrouz R, Topel CH, Misra V, Pomero F, Giraudo $A$, et al. Hypoalbuminemia, systemic inflammatory response syndrome, and functional outcome in intracerebral hemorrhage. Journal of critical care. 2017;41:247-53.

23. Prabhu G, Raadha A, Balasubramaniyan S, Avinash A, Nikhitha G, Anuradha A. Study of anaemia as an individua risk factor in CVA: ischemic stroke. J Evolution Med Dental Sci. 2015;4(59):10351-6.

24. Murray CJ, Lopez AD. Alternative projections of mortality and disability by cause 1990-2020: Global Burden of Disease Study. Lancet. 1997;349(9064):1498-504.

25. Cucchiara BL, Kasner SE, Wolk DA, Lyden PD, Knappertz VA, Ashwood T, et al. Early impairment in consciousness predicts mortality after hemispheric ischemic stroke. Crit Care Med. 2004;32(1):241-5.
26. Feigin VL, Lawes CM, Bennett DA, Anderson CS. Stroke epidemiology: a review of population-based studies of incidence, prevalence, and case-fatality in the late 20th century. Lancet Neurol. 2003;2(1):43-53.

27. Naghavi M, Abajobir AA, Abbafati C, Abbas KM, Abd-Allah F, Abera SF, et al. Global, regional, and national age-sex specific mortality for 264 causes of death, 1980-2016: a systematic analysis for the Global Burden of Disease Study 2016. Lancet. 2017;390(10100):1151-210.

28. Feigin VL, Forouzanfar MH, Krishnamurthi R, Mensah GA Connor M, Bennett DA, et al. Global and regional burden of stroke during 1990-2010: findings from the Global Burden of Disease Study 2010. Lancet. 2014;383(9913):245-55.

29. Belayev L, Zhao W, Pattany PM, Weaver RG, Huh PW, Lin $B$, et al. Diffusion-weighted magnetic resonance imaging confirms marked neuroprotective efficacy of albumin therapy in focal cerebral ischemia. Stroke. 1998;29(12):2587-99.

30. Dziedzic T, Pera J, Slowik A, Gryz-Kurek EA, Szczudlik A. Hypoalbuminemia in acute ischemic stroke patients: frequency and correlates. Eur J Clin Nutr. 2007;61(11):131822.

31. Franch-Arcas G. The meaning of hypoalbuminaemia in clinical practice. Clin Nutr. 2001;20(3):265-9.

32. Ginsberg MD. Neuroprotection for ischemic stroke: past, present and future. Neuropharmacology. 2008;55(3):363-89.

33. Alvarez-Perez F, Castelo-Branco M, Alvarez-Sabin J. Albumin level and stroke. Potential association between lower albumin level and cardioembolic aetiology. Intern $\mathrm{J}$ Neuroscience. 